\title{
Space-Wise approach for airborne gravity data modelling
}

\author{
D. Sampietro1 ${ }^{1}$ - M. Capponi ${ }^{2}$ - A. H. Mansi $^{2}$ - A. Gatti ${ }^{2}$ - P. Marchetti ${ }^{3}$ \\ F. Sansò ${ }^{1,2}$
}

Received: 23 August 2016 / Accepted: 2 December 2016 / Published online: 18 December 2016

(C) Springer-Verlag Berlin Heidelberg 2016

\begin{abstract}
Regional gravity field modelling by means of remove-compute-restore procedure is nowadays widely applied in different contexts: it is the most used technique for regional gravimetric geoid determination, and it is also used in exploration geophysics to predict grids of gravity anomalies (Bouguer, free-air, isostatic, etc.), which are useful to understand and map geological structures in a specific region. Considering this last application, due to the required accuracy and resolution, airborne gravity observations are usually adopted. However, due to the relatively high acquisition velocity, presence of atmospheric turbulence, aircraft vibration, instrumental drift, etc., airborne data are usually contaminated by a very high observation error. For this reason, a proper procedure to filter the raw observations in both the low and high frequencies should be applied to recover valuable information. In this work, a software to filter and grid raw airborne observations is presented: the proposed solution consists in a combination of an along-track Wiener filter and a classical Least Squares Collocation technique. Basically, the proposed procedure is an adaptation to airborne gravimetry of the Space-Wise approach, developed by Politecnico di Milano to process data coming from the ESA satellite mission GOCE. Among the main differences with respect to the satellite application of this approach, there is
\end{abstract}

\footnotetext{
D. Sampietro

daniele.sampietro@g-red.eu

1 GReD s.r.l. c/o ComoNExT, Via Cavour 2,

22074 Lomazzo, CO, Italy

2 DICA, Politecnico di Milano, Piazza Leonardo da Vinci 32, 20133 Milano, MI, Italy

3 Eni s.p.a., via Emilia 1, 20097 San Donato Milanese, MI, Italy
}

the fact that, while in processing GOCE data the stochastic characteristics of the observation error can be considered a-priori well known, in airborne gravimetry, due to the complex environment in which the observations are acquired, these characteristics are unknown and should be retrieved from the dataset itself. The presented solution is suited for airborne data analysis in order to be able to quickly filter and grid gravity observations in an easy way. Some innovative theoretical aspects focusing in particular on the theoretical covariance modelling are presented too. In the end, the goodness of the procedure is evaluated by means of a test on real data retrieving the gravitational signal with a predicted accuracy of about $0.4 \mathrm{mGal}$.

Keywords Gravity · Airborne gravimetry · Removecompute-restore procedure - Covariance modelling . Space-Wise approach

\section{Introduction}

The improvements in satellite geodesy and Earth's global gravity models (GGM) of the last years have nowadays opened the way for important applications such as accurate determination of the actual shape of the Earth, i.e. the geoid (Sansó and Sideris 2013), or global crust-mantle boundary determination (Reguzzoni and Sampietro 2015; Boumann et al. 2015; Mariani et al. 2013), post-glacial rebound (Vermeersen 2003), and geostrophic currents modelling (Knudsen et al. 2011; Gilardoni et al. 2013; Menna et al. 2013). However, applications such as resource exploration still demand a much higher resolution than the one obtained by satellite-only observations and a much higher accuracy than that obtained from GGM. 
On the one hand, even data from the ESA GOCE satellite mission (Drinkwater et al. 2003), one of the most important gravity missions of the last years which had homogeneously observed the Earth gravitational field exploiting direct satellite gradiometry, have been able to resolve the gravitational field with accuracies better than $1 \mathrm{mGal}(1 \mathrm{mGal}=$ $10^{-5} \mathrm{~m} / \mathrm{s}^{2}$ ) at a spatial resolution of about $100 \mathrm{~km}$ (Brockmann et al. 2014; Voigt and Denke 2015). As well known, this is not enough for exploration geophysics where similar, or even better, accuracies are required up to a spatial resolution of about $1 \mathrm{~km}$ (Hinze et al. 2013).

On the other hand, also the most accurate GGM, such as EGM2008 (Pavlis et al. 2012), contains non-homogeneous terrestrial datasets that are reflected in biases and distortions in the final gravity field model (Gatti et al. 2013). Even the integration of GOCE data in high-degree GGM, as in the EIGEN-6C4 (Förste et al. 2014) or in the GECO model (Gilardoni et al. 2016) solves the problem only partially, leaving unchanged possible inconsistencies of the GGM within the medium high frequencies. As a consequence, for resource exploration purposes, other means have to be found to resolve high frequencies, which can potentially provide important information on the upper crustal structure. Observations collected closer to the Earth's surface, such as airborne, shipborne and terrestrial gravity data, represent attractive complementary information to estimate these high frequencies. However, these measurements are limited in their spatial extent and, due to the characteristics of modern gravimeters, can contain systematic errors in the low frequencies. However, at the same time, they can significantly contribute to the determination of the medium to high frequencies of the gravity field. Airborne gravimetry and shipborne gravimetry are of particular interest to resolve these frequencies since they provide gravity measurements in a cost-effective way. Of course, solving these medium to high frequencies of the gravity field can give important benefits also to other applications, such as local and regional geological studies (e.g. Bell et al. 1999) or geoid determination (e.g. Forsberg et al. 2000). Nevertheless, in the present work we will mainly focus on exploration geophysics applications. For further details on airborne and shipborne gravimetry and their characteristics, the interested reader can refer to the literature (Schwarz and Li 1996, 1997; Glennie et al. 2000; Bruton 2000; Térmens and Colomina 2005). We would just mention here the fact that typical aero-gravimetric data for geophysical exploration cover areas of around $100 \mathrm{~km} \times 100 \mathrm{~km}$ (see for instance CarbonNet Project 2012), so that significant gravimetric data are contained in the observations with a spatial resolution of about $60 \mathrm{~km}$. The standard geophysical processing algorithms address in fact wavelengths between 1 and $50 \mathrm{~km}$, or even less.

In the following, an analysis of the prediction of a grid or a set of filtered along-track gravity anomalies obtained by merging GGM and airborne dataset, based on a combination of along-track Wiener filter and Least Squares Collocation adjustment, is presented. In this framework, we will call "processing" the calculations done to convert "raw aerogravimetric data" into a set of predicted gravity disturbances or gravity anomalies. The "raw aero-gravimetric data" is, in this contest, the accelerations resulting from direct gravimetric measurements minus inertial accelerations derived from GNSS (Global Navigation Satellite System) and IMU (Inertial Measurement Unit) data as well as all the other deterministic effects, such as the Eötvös (Harlan 1968) and the lever arm effects (Saint-Jean et al. 2007). The result is a sequence, along the aircraft trajectory, of the vertical component (referring to a terrestrial reference system) of the gravity plus all the biases and random errors inherited from the gravity measurements and the inertial data processing.

The topic of airborne gravimetry "processing" has been tackled and solved in several ways in geodetic literature, here we mention first the Calgary approach (Schwarz et al. 1990) and the collocation approach (Tscherning et al. 1998), particularly interesting to change from the observed functional of the anomalous gravitational potential to another predicted functional. The main problems to be faced in filtering airborne observations, as always with gravity, are to disentangle the long wavelength signal from the observation coloured noise and to properly filter the high frequencies. The former can be tackled, nowadays, by exploiting information derived from satellite gravimetry/gradiometry and radar altimetry combined with surface data in GGMs. In the following, a solution of what we call "processing", i.e. basically a filtering and gridding procedure, is presented.

\section{Theoretical aspects}

As pointed out in the introduction, the problem of filtering raw airborne gravity observations consists basically in the separation of the observation coloured noise from the actual gravitational signal. In the present work, we propose a solution scheme, for this issue, similar to the one used in the GOCE Space-Wise solution (Reguzzoni and Tselfes 2009), but adapted of course to the airborne gravimetry. Among the main differences with respect to the GOCE Space-Wise solution, we would like to point out the fact that while in the case of GOCE, the gradiometer observation error had been studied for a long time and therefore it can be considered well known (Smit et al. 2000) (at least in terms of spectral properties), in the case of airborne gravimetry the gravimeter observation error is usually unknown. Consequently, it should be recovered from the observations themselves and from the knowledge of the actual gravitational field. Another difference is that for the official GOCE solutions the final 
product should be independent from external data, while for airborne data processing this is not required.

The implemented procedure that processes raw aerogravimetric data in order to get a grid of predicted gravity disturbances (or along-track filtered observations) can be summarized as follows:

1. first a reference signal, called $\delta g_{\text {ref }}$ is created along one track from existing global models (e.g. EIGEN-6C4 or GECO including GRACE and GOCE products) adding to it the residual terrain correction (RTC):

$\delta g_{\text {ref }}=\delta g_{\mathrm{GGM}}+\delta g_{\mathrm{RTC}}$

where $\delta g_{\mathrm{GGM}}$ is the spherical harmonic synthesis of the GGM up to its maximum degree/order $L_{\max }$ computed along the aircraft trajectory, while $\delta g_{\mathrm{RTC}}$ is the RTC computed on the same points. Terrain corrections are computed, exploiting fast Fourier transform algorithms, by means of the GTE software (Sampietro et al. 2016). Note that in order to perform the RTC the digital elevation model is smoothed using a moving average window, with a spatial resolution that depends on $L_{\max }$, given by Lambeck (1990):

$$
\lambda=\frac{40.000 \mathrm{~km}}{L_{\max }+0.5} .
$$

2. We subtract the contribution of the global model used to generate the reference signal itself up to some intermediate degree $L_{\text {cut }}$ (e.g. 360) from the observations of the considered track $\delta g_{\text {obs }}$, as well as from $\delta g_{\text {ref }}$. Moreover, the effect of a coarse global terrain correction (from the same degree $L_{\text {cut }}$ ) is removed too, by exploiting global gravity field model related to topography such as $\mathrm{dV}_{\text {_- }}$ ELL_RET2012 (Hirt et al. 2012). If required, a linear trend for each reduced flight line is also removed. This operation is justified by the fact that the gravimeter observations, and more in general the inertial observations, are usually contaminated by drifts and biases (Hamilton and Brul 1967). The above procedure represents our remove step that leaves the reduced observations $\delta g_{\text {obs }}^{\text {red }}$ and the reduced reference signal $\delta g_{\text {ref }}^{\text {red }}$ with zero mean, smaller amplitude and spatial correlation with respect to the original ones.

3. $\delta g_{\text {obs }}^{\text {red }}$ are now Wiener-filtered track-by-track and the linear trend coming from the reference signal is added back, thus obtaining a number of regularly sampled tracks of the filtered residual gravity disturbances $\delta g_{\text {fil }}^{\text {red }}$;

4. an analysis of the residuals at the crossovers, i.e. at the intersection between two different tracks, is performed, thus obtaining an empirical estimate of the noise stochastic properties along the aircraft track of $\delta g_{\text {fil }}^{\text {red }}$;
5. the signal covariance function is then estimated and modelled as a linear combination of a set of Bessel functions of the first order and zero degree (Watson 1995). This step can be performed automatically either by means of an innovative algorithm in the frequency domain, exploiting the well-known relation between 2D power spectral densities and Bessel functions (Kreh 2012), or by means of a non-negative Least Squares adjustment (Lawson and Hanson 1974);

6. a grid of $\delta g_{\text {obs }}^{\text {red }}$ (or a set of along-track filtered data) and a grid of its corresponding errors are generated by a Least Squares Collocation solution;

7. finally, the signals removed at point 2 are restored back, thus obtaining the desired grid.

The whole procedure is summarized in Fig. 1, while more details on each step are discussed in the following.

As it can be notice from the presented procedure, the upward/downward continuation of the reduced gravitational field has been neglected here. This is justified by a numerical experiment performed in the Alpine area $\left(8^{\circ} 30^{\prime} \mathrm{E}-9^{\circ} 30^{\prime} \mathrm{E}, 45^{\circ} 30^{\prime} \mathrm{N}-46^{\circ} 30^{\prime} \mathrm{N}\right)$. Basically, we simulated the upward/downward continuation of the reduced field by performing a residual terrain correction (with a signal amplitude of about $\pm 50 \mathrm{mGal}$ ) of the region on the DTM surface and at different altitudes. Results show that for height differences smaller than $1000 \mathrm{~m}$ neglecting the upward/downward continuation entails errors of the order of $0.5 \mathrm{mGal}$ (standard deviation). Note that the Alpine area, considered in this simple test, is among the most extreme regions (from the topographical point of view) of the world and therefore less dramatic conditions are in general investigated for exploration purposes (see for instance the real airborne acquisition presented in Sect. 3) thus justifying, at least numerically, the proposed procedure.

\subsection{The Wiener filter}

The first step of the whole procedure consists in computing the reference signal $\delta g_{\text {ref }}$, i.e. a signal as close as possible to the actual field without using the airborne data. This can be done by means of a high-degree global model (such as EIGEN-6C4 or GECO model) up to the its maximum degree/order (e.g. 2190) and adding the missing high frequencies by summing a residual terrain correction contribution. Practically, $\delta g_{\text {ref }}$ will be used to retrieve the low frequencies of the final filtered field (using a GGM that in principle fully exploits GRACE as well as GOCE data, this low frequencies are expected to be more reliable or at least better sampled than those derived from airborne observations) and to estimate the stochastic properties of the residual field. After that, both the observations and the reference signal are reduced for the low and medium-high frequencies. 
Fig. 1 Flow chart of the procedure implemented to filter and grid raw aero-gravimetric data (here it is assumed that the signal covariance is estimated from observation points)

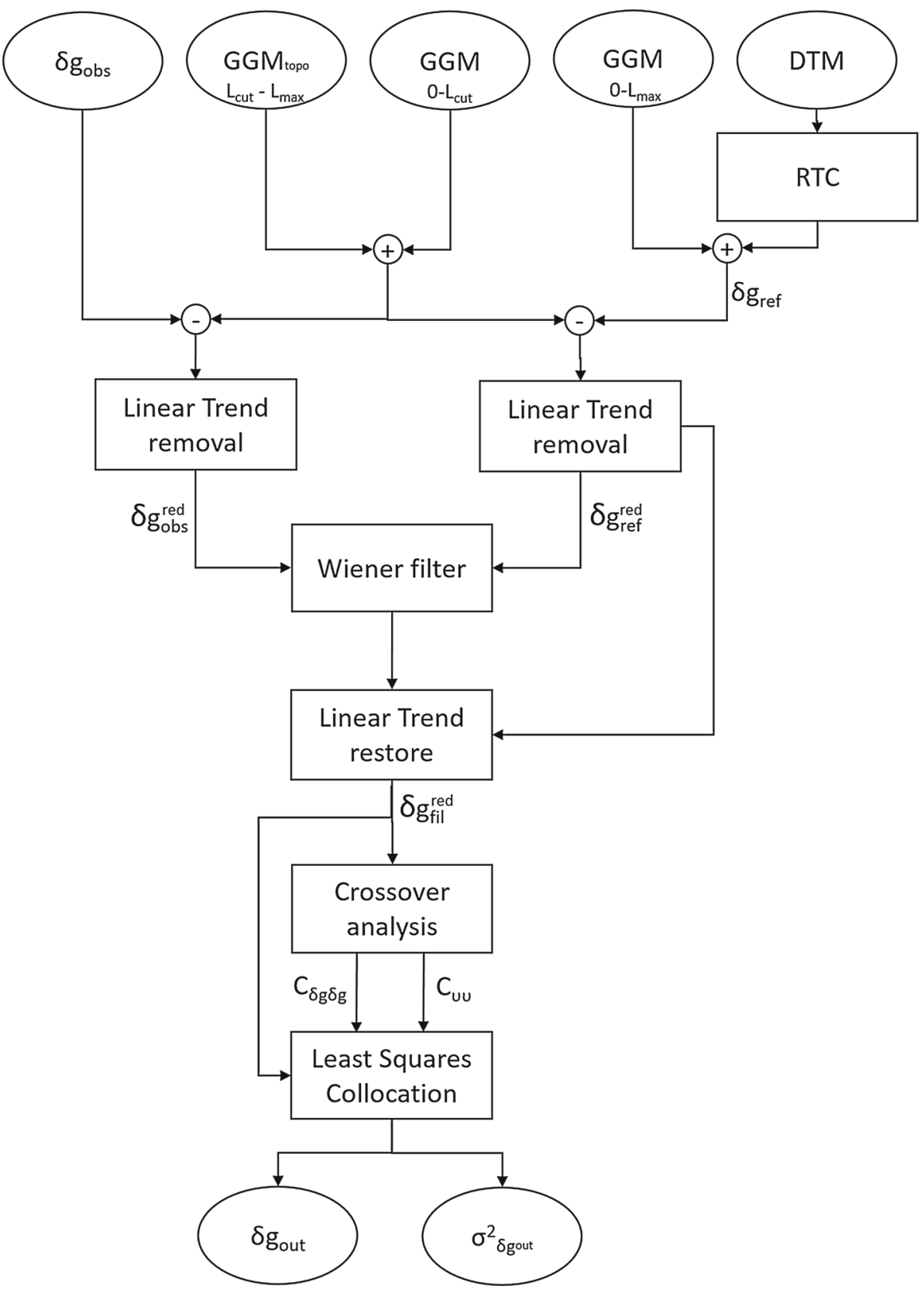

The former is done by subtracting the synthesis of the low harmonic degrees (up to a certain degree $L_{\text {cut }}$ for instance equal to 360) of the same model used to generate the reference signal itself, while the latter is fulfilled by subtracting the synthesis of the high degrees of a global model related to topography (e.g. dV_ELL_RET2 012).

This operation will leave a reference signal with zeroed low frequencies and reduced medium-high frequencies. The linear trend is also removed from the reference signal as well as from the observations. Once the data have been reduced, the track-by-track Wiener filter, $G$, in the frequency domain can be applied (Wiener 1949). Namely, the filter is computed as:

$$
G=\left(\frac{S_{\delta g}}{S_{\delta g}+S_{v}}\right)^{\frac{1}{2}}
$$

where $S_{\delta g}$ and $S_{v}$ are the power spectral densities of the reduced signal and the observation noise, respectively. The former is simply computed from the reduced reference signal $\delta g_{\text {ref }}^{\text {red }}$, while the denominator is retrieved from the reduced observations:

$$
\begin{aligned}
& S_{\delta g}=\left|\delta \hat{g}_{\text {ref }}^{\text {red }}\right|^{2} \\
& S_{\nu}=\left|\delta \hat{g}_{\text {obs }}^{\text {red }}\right|^{2}-S_{\delta g}
\end{aligned}
$$


where $\hat{\bullet}$ represents the Fourier transform of the quantity $\bullet$. Note that, since the reference signal does not contain the low frequencies, $S_{\delta g}$ will be 0 up to a certain frequency implicitly defined by $L_{\text {cut }}$. Consequently, the Wiener filter will replace the low frequencies of the airborne gravity data (which are known to be at least bad sampled) with the GGM ones, which, as said before, are expected to be more reliable. The only open point is how to choose the proper $L_{\text {cut }}$; however, it can been empirically fixed on the basis of the formal GGM error, e.g. by selecting $L_{\text {cut }}$ in such a way that the GGM cumulative error is smaller than a given threshold or as a function of the dimension of the investigated area (e.g. up to degree 360 for regions of about $1^{\circ} \times 1^{\circ}$ like in the numerical test reported in Sect. 3). Finally, after the Wiener filtering, the linear trend of each track, estimated from the reduced reference signal, is added back, obtaining as output a number of regularly sampled tracks of the reduced gravity disturbances $\delta g_{\text {fil }}^{\text {red }}$. Note that, within this filtering, the topography is used for two different independent purposes: it is in fact used in order to estimate the high frequency part of the signal spectrum $S_{\delta g}$ and also to reduce the amplitude of the signal at intermediate frequencies in a remove-restore fashion. While in the former, as already stated, it is computed by means of high-resolution local residual terrain effect calculation, basically trying to add to the reference model the very high frequencies (i.e. from degree $L_{\max }$ to $+\infty$ ), in the latter it is computed from a global model (from degree $L_{\text {cut }}$ to degree $L_{\max }$ ).

\subsection{Crossover analysis}

At this point, a crossover analysis is performed to retrieve information on the $\delta g_{f i l}^{r e d}$ error. It should be observed that, in principle, it is possible to compute the spectrum of the estimation error of the filtered observations just propagating those of the observation error within the Wiener filter as:

$S_{\epsilon_{\delta g}}=G^{2} S_{v}$

However, since both the filter $G$ and the error spectrum $S_{v}$ depend on the GGM used, we prefer to directly consider the filtered data and evaluate their accuracies empirically. Basically, this is done by means of the crossover analysis. Note that in the classical airborne gravity data filtering (see for instance the technical documentation related to the dataset used for the numerical test CarbonNet Project 2012) a crossover adjustment, in which low frequencies of the gravitational field are retrieved on the basis of the crossovers, is usually performed. In our analysis, we use crossovers, as already stated, only to empirically retrieve the along-track observation error covariance.

In details, this analysis consists of modelling each aircraft track as a straight line, which parameters are estimated by means of Least Squares Adjustments, and subsequently

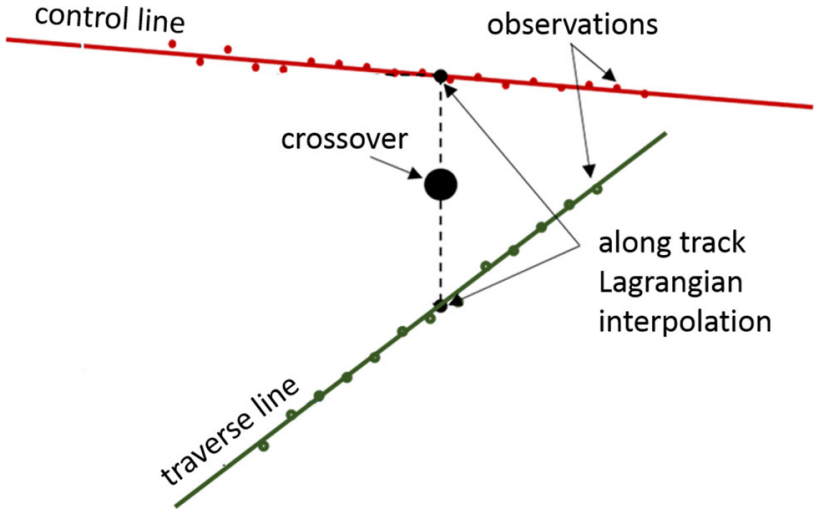

Fig. 2 Geometry of the crossover analysis

computing the gravitational field at the intersections of each couple of lines. For this purpose, the flight lines are divided, according to their flying direction, into two sets: namely traverse lines and control lines. For each crossover, i.e. for each intersection between a traverse and a control line, we separately interpolate the gravity field from the traverse line only and from the control line only (see Fig. 2).

This is done by firstly applying a Lagrangian interpolation (Davis 1975) to predict the gravity value on the modelled line in correspondence to the intersection point and, after that, by moving the estimated values in the vertical direction. This last step is performed by linearizing the problem and computing the second radial derivative of the gravity field from the reference model. It should be observed that the effect of both these "movements" is, in general, quite limited. In fact, considering for instance the real acquisition treated in the next section, the maximum along-track shift results to be smaller than $4 \mathrm{~m}$, while in the vertical direction we found a maximum distance between two tracks at the crossover of only $1.5 \mathrm{~m}$, which is negligible when dealing with the reduced signal.

The above procedure allows to estimate a set of differences between the gravitational field at the same observed point from couples of different tracks or, in other terms, it allows to evaluate on a set of points the error of the filtered gravity disturbances. We consider for each control line the whole set of estimated errors at the crossovers and compute the empirical along-track error covariances. Assuming that the along-track error is stationary, we can average the error covariances of each single control line and finally estimate a theoretical along-track error covariance function. Finally, this error covariance can be interpolated by means of a proper theoretical covariance function $C_{v v}(\psi)$ (where $\psi$ is the spherical distance along the aircraft track) to be used in the subsequent Least Squares Collocation.

This crossover analysis does not only permit to model the error covariance function $C_{\nu v}(\psi)$ but also allows to have 
some indications on the goodness of the previous Wiener filtering procedure.

\subsection{Covariance function estimate and least squares collocation}

In order to compute the final grid (or the final $\delta g$ along track), the covariance function of the reduced gravitational signal is required. In the proposed solution, this information can be retrieved in two different ways. In the former method, a grid of $\delta g_{\text {ref }}$ values at a constant height is generated from the initial reference global gravity model. The reference reduced grid of $\delta g_{\text {ref }}^{\text {red }}$ is used to generate the empirical covariance function, which is fit with a set of $n$ Bessel functions of the first order and zero degree (Kreh 2012). The covariance function is built as a linear combination (with positive coefficients) of these Bessel functions. The fitting step is performed automatically in the frequency domain exploiting the well-known relation between the 2D power spectral densities and Bessel functions (Watson 1995). Details on the theoretical aspects related to this procedure are reported in "Appendix 1".

The latter method to model the signal covariance function consists in empirically computing it from the along-track filtered reduced signal and subsequently modelling it with a linear combination of Bessel functions, coefficients of which are estimated by means of a non-negative Least Squares Adjustment. The main differences between the two solutions are that while the former estimates the covariance from external data, the latter uses only the filtered airborne signal. In both methods, the empirical covariance can be zeroed for distances larger than a certain threshold avoiding the covariance function to have significant oscillations and simplifying the Least Squares Collocation.

Whatever method is chosen, once the theoretical covariance function is estimated it can be used to build the covariance matrix required in order to compute the final result. Note that at this stage we have a set of along-track filtered and reduced observations $\delta g_{\text {fil }}^{\text {red }}$ characterized by having independent (line by line) and stationary noise with known stochastic characteristics retrieved by the crossovers analysis and a spatially correlated signal due to the gravitational field only. In this step, the combination between the GGM grid and the along-track aero-gravimetric data can be easily performed if an estimate of the GGM error is available. Finally, in order to obtain the full gravitational signal, all the frequencies removed before the Least Square Collocation adjustment are restored.

\section{Numerical test}

The algorithm presented in the previous sections has been implemented into a software called SWAG (Space-Wise for

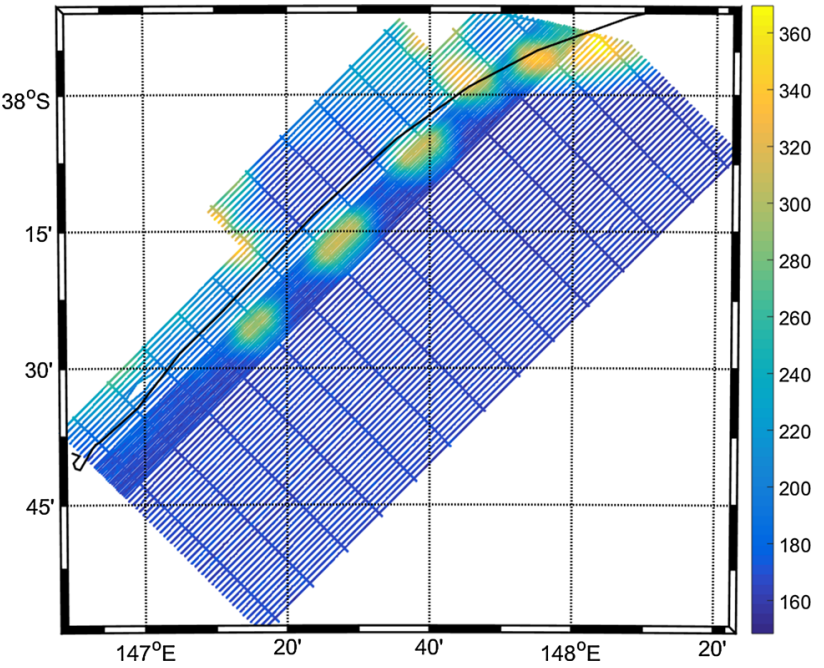

Fig. 3 Along-track flight altitude (the turns of the aircraft are omitted for the sake of readability). Black line represents the coastline; unit (m)

Airborne Gravity) and tested on a real airborne acquisition performed in the framework of the CarbonNet Project (2012). The dataset is made of 404384 aero-gravimetric observations acquired in 2011 by Sander Geophysics Ltd. (SGL) to provide a better understanding of the onshore, nearshore and immediate offshore geology of the Gippsland Basin, a sedimentary basin situated in south-eastern Australia, about $200 \mathrm{~km}$ east of the city of Melbourne. The survey was conducted using SGLs AIRGrav system mounted on a Cessna Grand Caravan 208B. In details, more than $10000 \mathrm{~km}$ length of data were acquired along flight lines oriented northeast and southwest with $1 \mathrm{~km}$ line spacing. Moreover, a $9 \mathrm{~km}$ wide strip along the coast was flown at $500 \mathrm{~m}$ line spacing. Tie lines were flown on the northwest southeast direction at $10 \mathrm{~km}$ line spacing. The survey was flown at an average ground speed of about $50 \mathrm{~ms}^{-1}$ at an altitude of 160-170 m offshore and with a minimum clearance of $305 \mathrm{~m}$ above towns (see Fig. 3 where the flight tracks, the turns of the aeroplane, as well as flight altitude are shown). The dataset includes, among the others, unfiltered raw-gravitational observations as well as $50 \mathrm{~s}$ (corresponding to about $2.5 \mathrm{~km}$ ) half wavelength filtered free-air anomaly sampled at $2 \mathrm{~Hz}$ (corresponding to about $25 \mathrm{~m}$ ).

All the tests have been performed on a single node of a supercomputer equipped with two 8-cores Intel Haswell $2.40 \mathrm{GHz}$ processors (for a total of 16 cores) with $128 \mathrm{~GB}$ RAM.

In the current numerical test, the along-track raw gravity observations are computed by SGL by subtracting the GPSderived aircraft accelerations from the inertial ones, applying the Eötvös correction and removing the normal gravity effect. As expected, the anomalous gravitational signal is largely dominated by the noise. It can also be observed that the 
noise has both low and high frequencies components, thus making the filtering process a complex problem. Moreover, as stated in the Introduction, no information on the stochastic characteristics of the observation noise is available. As explain in Sect. 2, the first step of the developed filtering procedure consists in computing the reference signal on the 404384 observation points. This is done by synthesizing the gravity disturbance from the GECO model up to its maximum degree/order (i.e. $L_{\max }=2190$ ), and adding to this quantity a residual terrain correction. The former is done by an ad-hoc software developed in parallel $\mathrm{C}$ language. The computation of this harmonic synthesis up to degree and order 2190 on the 404384 points takes about $20 \mathrm{~min}$; however, the software is able to properly down-sample the observations according to the GGM maximum degree and the distance between two points on the same line, thus reducing the computational time up to 10 times. As for the latter, i.e. the residual terrain correction, we used, as said before, the GTE software (Sampietro et al. 2016). In details, the residual terrain correction is computed as the difference between a full-resolution digital elevation model (Whiteway 2009) and a smoothed elevation model obtained by applying a moving average window, according to Eq. 2, of about $20 \mathrm{~km}$ to the full-resolution one. The second step of the filtering procedure consists in reducing, both the observations and the reference signal, for the low frequency, which are known to be very well estimated in the GECO model due to the combination of EGM2008 (containing GRACE data) and GOCE observations, while they are badly retrievable from the airborne gravimetric data. This is done again by synthesising the gravity disturbances up to a maximum degree and order of $L_{\text {cut }}=360$ (corresponding to a wavelength of about $100 \mathrm{~km}$ ) using the GECO model and synthesising the medium-high frequencies in the range between $L_{\text {cut }}=360$ and $L_{\max }=2190$ using dV_ELL_RET2012 model. As an example, some details of the Wiener filtering of one flight track (namely track number 204800) are reported in the following. From the computational point of view, the Wiener filtering, exploiting the properties of the FFT, requires about 1 second for each flight line for a total of less than $2 \mathrm{~min}$ for the whole dataset.

In Fig. 4 the observed gravitational field, together with the synthesis from the GECO model up to degree/order 2190 and the reference signal (i.e. the GECO derived signal plus the RTC), for line 204800 is shown. Again, it can be observed how the signal is completely dominated by the observations error which contains very powerful high frequencies but also trends and biases. In Fig. 5, the filtered reduced signal, once the linear trend has been restored, is compared to the reference one thus showing the improvements, due to the airborne observations, to the initial GGM-based reference signal. The order of magnitude of this improvement is of $2.65 \mathrm{mGal}$ (stan-

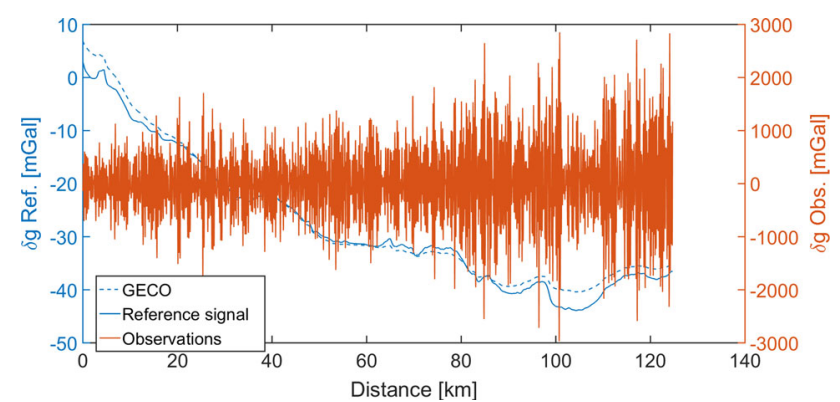

Fig. 4 Along-track reference, GMM and observed gravity disturbances for line 204800; unit (mGal)

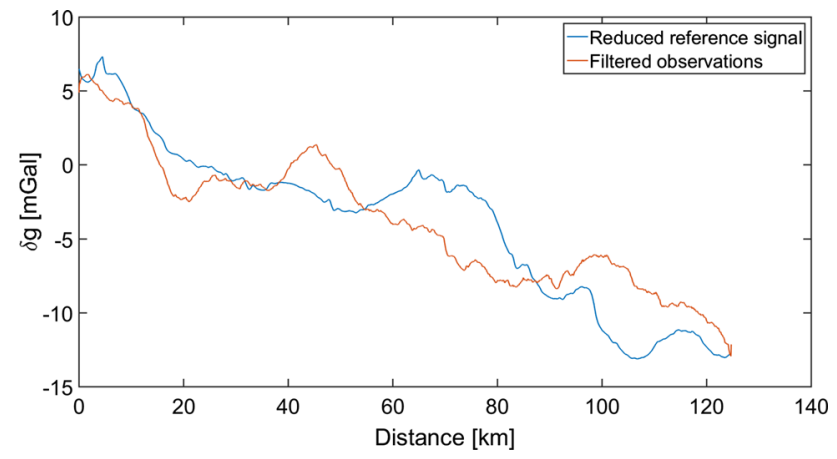

Fig. 5 Along-track reference and filtered gravity disturbances for line 204800; unit (mGal)

Table 1 Statistics of the residuals between different lines of the filtered gravity disturbances at the crossovers

\begin{tabular}{llll}
\hline Mean $(\mathrm{mGal})$ & $\mathrm{SD}(\mathrm{mGal})$ & Min $(\mathrm{mGal})$ & Max $(\mathrm{mGal})$ \\
\hline 0.036 & 1.01 & -2.83 & 4.80 \\
\hline
\end{tabular}

dard deviation), for the considered flight track, which is coherent with the expected accuracy of the GECO model.

After applying the Wiener filtering on the reduced signal, the crossover analysis is then applied. A total of 1479 crossovers has been identified and analysed by the algorithm described in Sect. 2.2 highlighting an along-track coloured noise with a standard deviation of $1.01 \mathrm{mGal}$ and a correlation length of about $2 \mathrm{~km}$ (statistics on the residuals obtained by the crossover analysis are reported in Table 1).

As for the covariance of the reduced signal, it has been computed by means of the two methods described in the previous section and it is shown in Fig. 6.

Actually, the main differences between the two covariances are due to the fact that the two methods use different inputs (filtered airborne along-track observations and grid of the reference model, respectively) and use also different regions to estimate the signal covariance. In order to compute the final result by means of the Least Squares Collocation algorithm, the dataset is down-sampled by a factor 
10 reducing the number of the observations to about 40000 points. This is justified by the fact that the initial dataset is sampled at $25 \mathrm{~m}$ and it is corrupted by a noise with approximate correlation length of about $2 \mathrm{~km}$. As a consequence, the contribution to the final estimate of points closer that $250 \mathrm{~m}$ can be assumed to be quite limited. The Least Squares Collocation algorithm, together with the covariance function estimation and modelling, takes about $45 \mathrm{~min}$ to compute the final gravitational field on the 404384 observation points.

The final results in terms of along-track reduced gravity disturbances, and their predicted errors are shown in Fig. 7, where it can be seen that the predicted error (referred to the data at flight altitude or to the grid at mean flight level) is of the order of $0.6 \mathrm{mGal}$, decreasing to only $0.4 \mathrm{mGal}$ when the distance between two any consecutive flight tracks is of $500 \mathrm{~m}$.

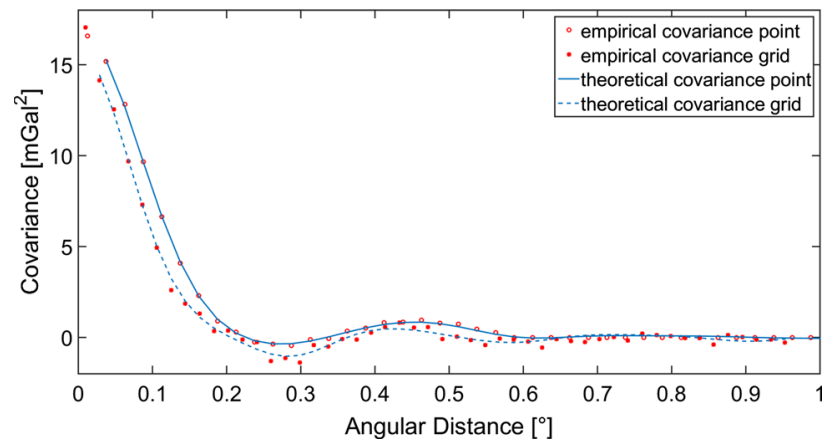

Fig. 6 Covariances; unit (mGal)
The proposed along-track solution has been compared with the published SGL solution funding differences with a standard deviation of $2.5 \mathrm{mGal}$ decreasing to $1.8 \mathrm{mGal}$ (see Fig. 8) when only the central part of region is considered (thus avoiding potential border effects). These differences are basically due to the difference in the low frequencies estimation (SGL used ground gravity benchmarks, while in the proposed solution low frequencies are retrieved by GOCE and GRACE satellites observations) and in the different high frequencies filtering (i.e. $3 \mathrm{~km}$ half wavelength filtered versus Least Squares Collocation). As for the border effect, it should be underlined that the proposed procedure, being based on a remove-restore procedure, greatly reduces, in the data reduction step, the border effect using information coming from GGM which is not the case in the SGL solution.

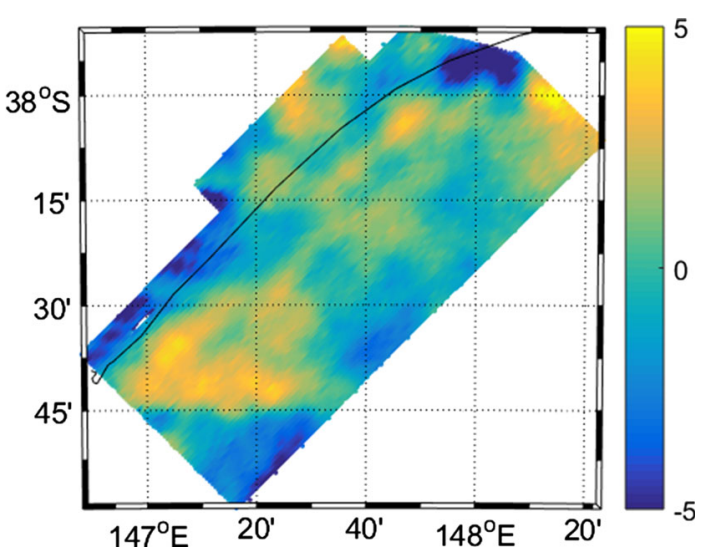

Fig. 8 Differences between the proposed and SGL ( $3 \mathrm{~km}$ half wavelength filtered) solutions; unit (mGal)
Fig. 7 Estimated corrections to the reference signal (left panels) and corresponding accuracies (rights panels) for gridded (up) and along-track points (down). Red dotted line represents the contour of the flight area; unit $(\mathrm{mGal})$
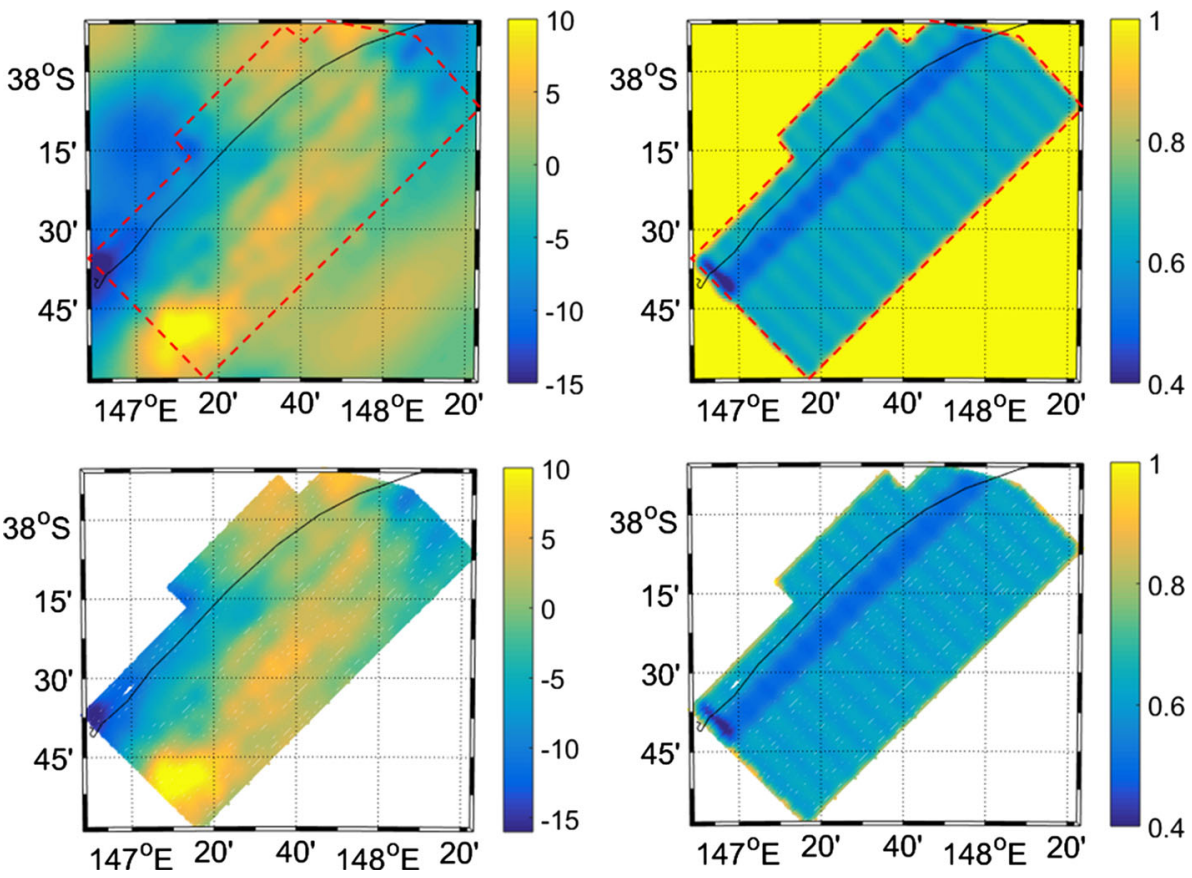


\section{Conclusions}

In this paper, a software, called SWAG, to filter and grid raw aero-gravimetric observation has been presented. The proposed solution is based on the classical remove-computerestore procedure and consists in a combination of a Wiener filter along the aircraft trajectory followed by a Least Squares Collocation adjustment for the prediction of grids (or set of points) of filtered gravity anomalies.

To solve the main problems related to the filtering of gravity observations, which are connected to the fact that the coloured observation noise is in general unknown when dealing with airborne gravimetry, a solution that exploits information from GGM has been developed. This solution allows to exploit both satellite and surface gravity data which are contained in the GGM to replace the low frequencies of the signal, which are known to be bad sampled by airborne gravimetry, and to stochastically separate the observation error from the expected gravitational signal.

As an indicator of the goodness of the Wiener filtering procedure and to estimate the error covariance function of the filtered observation, a crossover analysis has been developed too.

In order to use the Least Square Collocation to compute the final grid of gravity disturbances, an innovative method for the estimation of the covariance function, modelled with a linear combination of Bessel functions, has been applied.

The whole procedure has been thought for geophysical applications such as resource exploration, which require high accuracy and resolution not achievable with GGM and satellite-only data. A numerical tests on a real airborne dataset have been conducted to evaluate the goodness of the entire procedure implemented in SWAG software. The results show that the along-track Wiener filter allows to recover a signal with an accuracy of about $1 \mathrm{mGal}$ from observations characterized by a signal-to-noise ratio smaller than $1 \%$. Moreover, the subsequent Least Squares Collocation further filters the data exploiting the stochastic property of the residual gravitational field, which is suppose to be homogeneous and isotropic in the space, and those of the observation error which is considered isotropic and homogeneous along each single flight line but independent line by line. The whole procedure, applied on about 400000 points, takes about $120 \mathrm{~min}$ and gives a final predicted accuracy of the order of $0.6 \mathrm{mGal}$ decreasing up to $0.4 \mathrm{mGal}$ when the distance between two any consecutive flight tracks is of $500 \mathrm{~m}$.

Acknowledgements The authors would like to thank the management of Eni Upstream and Technical Services for the permission to present this work.

\section{Appendix 1}

The algorithm implemented to compute the covariance function from a grid of the reduced reference gravitational signal is briefly explained. We will suppose here to have a reduced grid $\delta g(\underline{x})$ with zero mean and with homogeneous and isotropic behaviours. Here, $\underline{x}$ represents the two planar coordinates of the grid. The first operation performed consists in computing the bi-dimensional fast Fourier transform $\delta \hat{g}(\underline{p})$ of $\delta g$, where $p$ represents the frequencies in the directions defined by the $(\underline{x})$ axis. We now consider $N$ bins, and for each bin $i$ we compute the following average:

$S_{i}(p)=\sum_{\underline{p} \in \Omega_{p_{i}}} \frac{|\delta \hat{g}(\underline{p})|^{2}}{n}$

where $p=|\underline{p}|, n$ is the number of values included within the $i$-th bin, and $\Omega_{p}$ is defined as:

$\Omega_{p_{i}}=\bar{p}_{i} \leq p \leq \bar{p}_{i}+\Delta p$

with $\bar{p}_{i}$ a set of values ranging from 0 to the maximum $p$ with an increment given by $\frac{\max |\underline{p}|}{N-1}$. Note that the final $S_{i}(p)$ is a step function that depends only on the radial coordinate $p$ of the plane $p$. It should be also observed that if we are interested in a covariance, which is a function on distances between points $r$ only, and this is always the case if the field is considered homogeneous and isotropic, then the covariance $C(r)$ can be simply inferred from the inverse Fourier transform of $S_{i}(p)$. Due to the relation between the bi-dimensional radially symmetric Fourier transform and Hankel transform, we have:

$S(p)=\int_{0}^{+\infty} \bar{J}_{0}(p r) C(r) \mathrm{d} r$

where the $\bar{J}_{0}$ function is related to the classical Bessel function of 0 order by the following relation:

$\bar{J}_{0}(p r)=2 \pi J_{0}(2 \pi p r)$

Of course $C(r)$ can be obtained by performing the inverse Henkel transform of eq. 9:

$C(r)=\int_{0}^{+\infty} \bar{J}_{0}(p r) S(p) \mathrm{d} p$. 
We shall also need the following formula (Watson 1995):

$$
\int_{0}^{+\infty} J_{0}(a r) \frac{J_{1}(b r)}{r} r \mathrm{~d} r=\left\{\begin{array}{ll}
0 & a>b \\
\frac{1}{b} & a<b
\end{array} .\right.
$$

If we consider $a=2 \pi p, b=2 \pi \bar{p}, \bar{J}_{0}(p r)=2 \pi J_{0}(p r)$, and $\bar{J}_{1}(p r)=2 \pi J_{1}(p r)$ we have:

$\frac{\bar{p}}{2 \pi} \int_{0}^{+\infty} \bar{J}_{0}(p r) \frac{\bar{J}_{1}(\bar{p} r)}{r} r \mathrm{~d} r=\left\{\begin{array}{ll}0 & p>\bar{p} \\ 1 & p<\bar{p}\end{array}\right.$.

Therefore, combining Eq. 11 and Eq. 13 we have:

$$
\begin{aligned}
& C(r)=\sum_{i=0}^{n} S_{i}\left[\frac{(i+1) \Delta p}{2 \pi} \frac{\bar{J}_{1}((i+1) \Delta r)}{r}\right. \\
& \left.-\frac{i \Delta p}{2 \pi} \frac{\bar{J}_{1}(i \Delta r)}{r}\right] .
\end{aligned}
$$

This allows to estimate a covariance function corresponding to a power spectrum described in terms of linear combination of step functions as a linear combination of $n$ Bessel functions of the first order and zero degree.

\section{References}

Bell RE, Childers VA, Arko RA, Blankenship DD, Brozena JM (1999) Airborne gravity and precise positioning for geologic applications. J Geophys Res Sol Earth 104(B7):15281-15292

Boumann J, Ebbing J, Meekes S, Fattah RA, Gradmann S, Bosch W (2015) GOCE gravity gradient data for litospheric modeling. Int J Appl Earth Obs 35:16-30

Brockmann JM, Zehentner N, öck E, Pail R, Loth I, Mayer Gürr T, Schuh WD (2014) EGM_TIM_RL05: an independent geoid with centimeter accuracy purely based on the GOCE mission. Geophys Res Lett 41(22):8089-8099

Bruton AM (2000) Improving the accuracy and resolution of SINS/DGPS airborne gravimetry, Phd Thesis, University of Calgary, Calgary Canada

CarbonNet Project Airborne Gravity Survey (2012) Gippsland Basin Nearshore Airborne Gravity Survey, Victoria, Australia. Victoria: Department of primary industries, Victoria State Government

Davis PJ (1975) Interpolation and approximation. Courier Corporation, New York

de Saint-Jean B, Verdun J, Duquenne H, Barriot JP, Melachroinos S, Cali J (2007) Fine analysis of lever arm effects in moving gravimetry. Int Assoc Geod Symp 130:809-816

Drinkwater MR, Floberghagen R, Haagmans R, Muzi D, Popescu A (2003) GOCE: ESA's first Earth Explorer Core mission. Space Sci Ser ISSI 17:419-432

Forsberg R, Olesen A, Bastos L, Gidskehaug A, Meyer U, Timmen L (2000) Airborne geoid determination. Earth Planets Space 52(10):863-866

Förste C, Bruinsma SL, Abrikosov O, Lemoine JM, Schaller T, Götze HJ, Ebbing J, Marty JC, Flechtner F, Balmino G, Biancale R (2014) EIGEN-6C4 The latest combined global gravity field model including GOCE data up to degree and order 2190 of GFZ Potsdam and GRGS Toulouse. In: Presented at the 5th GOCE User Workshop, Paris, 25-28 Nov 2014
Gatti A, Reguzzoni M, Sansó F, Venuti G (2013) The height datum problem and the role of satellite gravity models. J Geodesy 87(1):5-22

Gilardoni M, Menna M, Pulain PM, Reguzzoni M (2013) Preliminary analysis on GOCE contribution to the Mediterranean Sea circulation. ESA Spec Publ 722:206

Gilardoni M, Reguzzoni M, Sampietro D (2016) GECO: a global gravity model by locally combining GOCE data and EGM2008. Stud Geophys Geod 60(2):228-247

Glennie CL, Schwarz KP, Bruton AM, Forsberg R, Olesen AV, Keller $\mathrm{K}$ (2000) A comparison of stable platform and strapdown airborne gravity. J Geodesy 74(5):383-389

Hamilton AC, Brul BG (1967) Vibration-induced drift in LaCoste and Romberg Geodetic Gravimeters. J Geophys Res 72(8):2187-2197

Harlan RB (1968) Eotvos corrections for airborne gravimetry. J Geophys Res 73(14):4675-4679

Hinze WJ, Von Frese RR, Saad AH (2013) Gravity and magnetic exploration: principles, practices, and applications, 512. Cambridge University Press, New York

Hirt C, Kuhn M, Featherstone WE, Göttl F (2012) Topographic/isostatic evaluation of new-generation GOCE gravity field models. J Geophys Res Sol Earth 117(B5):B05407

Knudsen P, Bingham R, Andersen O, Rio MH (2011) A global mean dynamic topography and ocean circulation estimation using a preliminary GOCE gravity model. J Geodesy 85(11):861-879

Kreh M (2012) Bessel functions. Lecture notes, Penn StateGöttingen summer school on number theory

Lambeck K (1990) Aristoteles: an ESA mission to study the earth's gravity field. ESA J 14:1-21

Lawson CL, Hanson RJ (1974) Solving least squares problems, Englewood Cliffs. Prentice-Hall, Upper Saddle River

Mariani P, Braitenberg C, Ussami N (2013) Explaining the thick crust in Paran basin, Brazil, with satellite GOCE gravity observations. J S Am Earth Sci 45:209-223

Menna M, Poulain PM, Mauri E, Sampietro D, Panzetta F, Reguzzoni M, Sansó F (2013) Mean surface geostrophic circulation of the Mediterranean Sea estimated from GOCE geoid models and altimetric mean sea surface: initial validation and accuracy assessment. B Geofis Teor Appl 54(4):347-365

Pavlis NA, Holmes SA, Kenyon SC, Factor JK (2012) The development and evaluation of the Earth Gravitational Model 2008 (EGM2008), J Geophys Res-Sol Earth 117(B4):4406. doi:10. 1029/2011JB008916

Reguzzoni M, Sampietro D (2015) GEMMA: an Earth crustal model based on GOCE satellite data. Int J Appl Earth Obs 35:31-43

Reguzzoni M, Tselfes N (2009) Optimal multi-step collocation: application to the space-wise approach for GOCE data analysis. J Geodesy 83(1):13-29

Sansó F, Sideris MG (eds) (2013) Geoid determination: theory and methods. Springer, Berlin

Sampietro D, Capponi M, Triglione D, Mansi AHH, Marchetti P, Sansó F (2016) GTE: a new software for gravitational terrain effect computation: theory and performances. Pure Appl Geophys 173(7):1-19

Schwarz KP, Li Z (1997) An introduction to airborne gravimetry and its boundary value problems. Lecture notes in Earth sciences 65:312358

Schwarz KP, Sideris MG, Forsberg R (1990) The use of FFT techniques in physical geodesy. Geophys J Int 100(3):485-514

Schwarz KP, Li Y (1996) What can airborne gravimetry contribute to geoid determination? J Geophys Res 101(B8):873-881

Smit M, Koop R, Visser P, van den IJssel J, Sneeuw N, Muller J, Oberndorfer H (2000) GOCE End-to-End Performance Analysis, Final Report, ESTEC Contract No. 12735/98/NL/GD

Térmens A, Colomina I (2005) Network approach versus state-space approach for strapdown inertial kinematic gravimetry. Int Assoc Geod Symp 129:107-112 
Tscherning CC, Rubek F, Forsberg R (1998) Combining airborne and ground gravity using collocation. Int Assoc Geod Symp 119:18-23

Vermeersen LLA (2003) The potential of GOCE in constraining the structure of the crust and lithosphere from post-glacial rebound. Space Sci Rev 108(1-2):105-113

Voigt C, Denke H (2015) Validation of GOCE gravity field models in Germany. Newton's Bull 5:37-49
Watson GN (1995) A treatise on the theory of bessel functions. Cambridge University Press, Montpelier

Whiteway TG (2009) Australian bathymetry and topography grid. Geoscience Australia, Canberra

Wiener N (1949) Extrapolation, interpolation, and smoothing of stationary time series. MIT Press, Cambridge 